\title{
¿Conversion of the Knutson et al. Tropical Cyclone Climate Change Projections to Risk Model Baselines
}

\author{
STEPHEN JEWSON ${ }^{\mathrm{a}}$

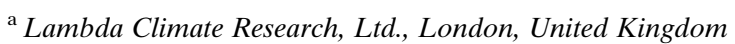

(Manuscript received 28 May 2021, in final form 3 October 2021)

\begin{abstract}
Knutson et al. recently published a metastudy that gives multimodel projections for changes in various properties of tropical cyclones under climate change. They considered frequency of tropical cyclones, frequency of very intense tropical cyclones, intensity of tropical cyclones, and total rainfall rate of tropical cyclones. For each of these properties, they reported changes globally and by basin for the six major tropical cyclone basins. The changes were presented as the change that would occur with $2^{\circ} \mathrm{C}$ warming of global mean surface temperature. These projections are potentially of great use to the tropical cyclone risk modeling community. However, most risk models use temporal baselines, such as the period from 1950 to 2019, and the Knutson et al. results can only be applied to risk models after some steps of adjustment involving past and future global mean surface temperature values. We derive the necessary adjustments and present and discuss some of the resulting projections, for different properties, basins, RCPs, and baselines. We find that the results are sensitive to the baseline being used, which implies that users of tropical cyclone risk models need to make sure they clearly understand what baseline their model represents before they adjust the model for climate change. One part of our analysis derives estimates of the implied impact of climate change so far on TC properties, relative to a representative baseline. The computer code we use to calculate the adjustments is available online.
\end{abstract}

SIGNIFICANCE STATEMENT: In a recent paper, Knutson et al. published multimodel projections for various properties of tropical cyclones under climate change. We show how to derive the adjustment factors required to put these results in a format that allows them to be incorporated into tropical cyclone risk models. We give various examples, including estimates of how climate change may already have changed tropical cyclones, relative to the behavior represented in risk models built using historical data. The computer code we have used to calculate the adjustment factors is available online.

KEYWORDS: Hurricanes/typhoons; Tropical cyclones; Interpolation schemes; Risk assessment

\section{Introduction}

Knutson et al. (2020, hereinafter K2020) give a detailed review of the state of understanding of the possible impacts of climate change on tropical cyclones (TCs). One part of the study involves a meta-analysis of a large number of previously published numerical modeling experiments from different authors. These experiments gave climate change responses for various different climate change scenarios. K2020 converted these responses to a common scenario, consisting of $2^{\circ} \mathrm{C}$ increase in global mean surface temperature (GMST), so that the results could be presented as distributions for this one scenario. In this way, K2020 derive multimodel estimates of the possible impacts of climate change for four specific properties of TCs: the frequency, the frequency of very intense storms, the intensity of storms, and the rainfall rate (see Figs. 1b, 2b, 3b, and 4 b in K2020, respectively, reproduced in Fig. 1 below). K2020 report changes in these properties globally, and separately for each of the six major TC basins. The distribution of results that

\footnotetext{
D Denotes content that is immediately available upon publication as open access.
}

Corresponding author: Stephen Jewson, stephen.jewson@ gmail.com arises from using multiple models is presented using five quantiles, the minimum, and the maximum.

These results are potentially extremely useful for the TC risk modeling community, that develops models that produce estimates of the probabilities of different levels of hazard and damage due to TCs (Vickery et al. 2000; James and Mason 2005; Emanuel et al. 2006; Hall and Jewson 2008; Yonekura and Hall 2011; Grieser and Jewson 2012; Asaduzzaman and Latif 2014; Lee et al. 2018; Sobel et al. 2019a; Arthur 2021). A major developer and user of these models is the insurance industry, in which the models are used for pricing and planning purposes. We describe these models in more detail in section 3. TC risk models are often used for understanding present-day risks and are typically based on estimates of the TC climate that are created using a combination of statistical analysis of historical observations, statistical simulation and numerical models. Different TC risk models focus on different aspects of TC behavior and impacts, but the most complete models create simulated tracks, wind fields, and rain, which they then use to generate estimates of surge depths and freshwater flood inundation. Simulated wind, rain, surge, and flood fields are converted into damage estimates using appropriate damage models based on engineering principles, damage simulations, and observed damages.

When TC risk models are used to help understand presentday risks, the climate represented in the models (i.e., the 
(a) TC Freq. Change $(+2 \operatorname{deg} \mathrm{C})$

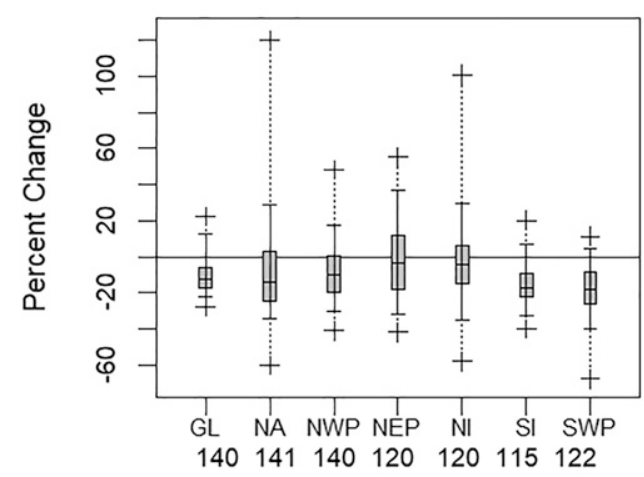

(c) TC Intensity Change (+ $2 \operatorname{deg}$ C)

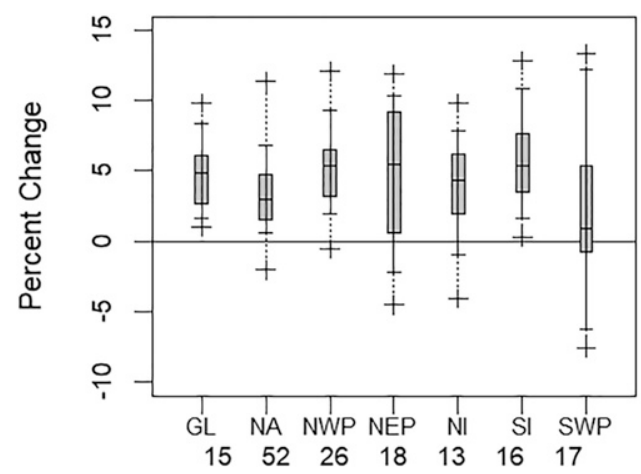

(b) Very Intense TC Freq. Change (+ 2 deg C)

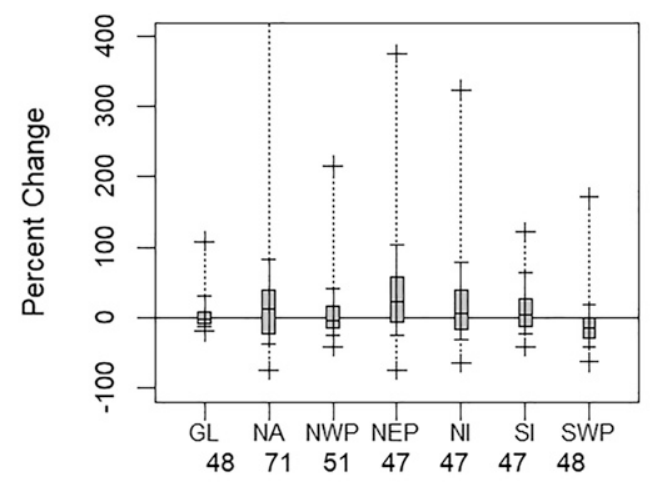

(d) TC Precip. Change (+ 2 deg C)

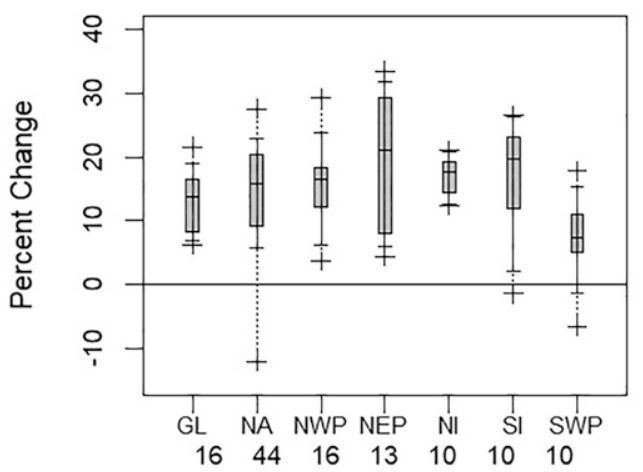

FIG. 1. Projections of changes in TC properties for a $2^{\circ} \mathrm{C}$ increase in GMST from K2020, as percent changes: (a) TC frequency, (b) the frequency of very intense TCs, (c) TC intensity, and (d) TC precipitation. Results are shown for seven regions: global (GL), North Atlantic (NA), Northwest Pacific (NWP), Northeast Pacific (NEP), North Indian Ocean (NI), South Indian Ocean (SI), and Southwest Pacific (SWP). For TC frequency, the box-andwhiskers show (from bottom to top) minimum, $5 \%$ quantile, $25 \%$ quantile, median, $75 \%$ quantile, $95 \%$ quantile, and maximum. For the other three variables, the box-and-whiskers show (from bottom to top) minimum, $10 \%$ quantile, $25 \%$ quantile, median, $75 \%$ quantile, $90 \%$ quantile, and maximum. The numbers below the horizontal axis show the number of models and scenarios used to derive the results for each region (in some cases, corrected relative to K2020).

number and properties of the TCs in the models) may be strongly influenced by the period of historical observations used to build the model. Modelers often use as many historical data as are reasonable given the accuracy of the data so as to increase the precision of parameter estimates and capture more historical examples of extremes. For instance, estimates of landfalling rates for North Atlantic hurricanes are often based on historical landfalling rates from HURDAT data (AOML 2014), using data from 1900 to the present, which is the period over which these data might be considered to be sufficiently accurate. A model built on data from 1900 to the present will represent the climate for the period 1900 to the present, in the absence of any intervention from the modelers to capture trends or variability in climate over time. We will refer to the period of historical data used to build a risk model as the model historical baseline. The question then arises as to whether models built using a historical baseline give a reasonable representation of the current climate. If the climate is stationary, then they may. If the climate is nonstationary, then they may or may not, depending on the trade-off between the benefit of using more data, and the potential bias caused by the differences between the climate of the model baseline and the current climate. Complicating matters, different parts of a model may be based on historical data from different periods of time. For instance, a North Atlantic Ocean hurricane model may be built using data from 1900 to the present to understand landfalling rates of hurricanes, but to understand the openocean behavior of hurricanes may be built on data from 1950 onward (reflecting the increase in data accuracy that occurred at that time due to the start of the use of aircraft reconnaissance) or from the mid-1960s onward (reflecting the further increase in accuracy that occurred due to the start of the use of satellite data). In these cases, for each parameter in the model it may nevertheless be possible to define an effective baseline, which defines the period of historical data that was most influential in determining the properties for that parameter in the 
model. We will assume that it is possible to determine such effective baselines in order to proceed with our analysis.

The K2020 multimodel projections of TC properties can potentially be used to adjust TC risk models that have been built using historical baselines. This would be a way to incorporate climate change into the models so that the models might be used to understand the impact of climate change on distributions of possible hazard and damage. However, since the K2020 results are presented as the change corresponding to a $2^{\circ} \mathrm{C}$ increase in GMST, while the risk models represent climate corresponding to specific historical baselines, the K2020 data cannot be applied to the risk models directly. Instead, one has to first adjust the K2020 results using interpolation and extrapolation, taking into account the baseline time period used to build the risk model, the period of time for which a climate projection is required, the climate scenario under consideration (e.g., which RCP; Meinshausen et al. 2011) and the changes in GMST between the baseline and projection periods. Once the K2020 data have been adjusted to represent the change relative to a historical baseline, for a given projection time and RCP, it can be applied to a risk model either by rebuilding the model or, more simply, by applying weights to the results from an existing model. In the latter case, probabilistic algorithms based on importance sampling have been developed for calculating the necessary weights (Jewson et al. 2019).

In this article we consider how the K2020 results can be adjusted using interpolation and extrapolation so that they can be applied to TC risk models. We derive some simple mathematical theory for making such adjustments, and present and discuss various results from applying the theory. We include some analysis of the implications for estimates of both climate change so far and future climate change, where by "climate change so far" we mean the impact of climate change that might be expected, today, relative to the historical baseline used in a risk model. For the insurance industry, understanding climate change so far is the most important aspect of climate change prediction, since understanding present-day risks is at the core of the insurance business and drives the pricing of most insurance contracts. The insurance industry is also interested in climate over $0-10-y r$ periods in the future for the purpose of planning, and $0-30$-yr periods in the future for the purposes of investment of assets. Other users of TC risk information would have a range of interests, from understanding present climate, to understanding long-term future climate (for long-term investors, and infrastructure projects with a long lifetime). We also compare the relative roles of different sources of uncertainty in the final results. We have made available in public archives the data from K2020, the GMST data, and various software tools for making the adjustments.

Before we proceed with our analysis, we note that extracting information about tropical cyclones from numerical models and applying it to TC risk models in this way is not the only way to incorporate information about climate variability and change into risk models. For instance, risk models can be built so that the properties of the tropical cyclones they simulate are inherently conditional on modes of climate variability (Hall and Jewson 2007; Yonekura and Hall 2011) or can be forced with large-scale fields from numerical climate models (Emanuel et al. 2006; Sobel et al. 2019a).

\section{Data}

Our analysis is based on two datasets: results from K2020, and GMST data for the past and future.

\section{a. K2020 data}

The K2020 data that we use consist of the 224 numbers that were used to generate Figs. 1b, 2b, 3b, and 4b in K2020 and are available at Jewson et al. (2021a). Of these numbers, 196 give projections for percent changes in four TC properties under $2{ }^{\circ} \mathrm{C}$ increase in GMST and 28 give sample sizes. The four properties are (i) TC frequency, where the definition of TC includes all storms with Saffir-Simpson categories from 1 to 5 , and additionally storms that have not reached category-1 (hurricane) intensity, but with maximum wind speeds of at least $17 \mathrm{~m} \mathrm{~s}^{-1}$ (i.e., tropical storm strength), sometimes referred to as category- 0 storms; (ii) the frequency of very intense TCs, where very intense means storms with SaffirSimpson categories of 4 or 5 ; (iii) the intensity of storms, where intensity is measured as maximum wind speed; and (iv) the precipitation rate for precipitation typically within $150 \mathrm{~km}$ of the storm center at a given time. For each of the four properties, the data give changes for seven regions: global (GL), North Atlantic (NA), northwest Pacific (NWP), northeast Pacific (NEP), north Indian (NI), south Indian (SI), and southwest Pacific (SWP). For each property and region, there are eight values. Seven are metrics that were derived by K2020 from the distribution of results from a large number of previous studies. For TC frequency, the seven metrics are minimum, $5 \%$ quantile, $25 \%$ quantile, median, $75 \%$ quantile, $95 \%$ quantile, and maximum. For the other three variables, the seven metrics are minimum, $10 \%$ quantile, $25 \%$ quantile, median, $75 \%$ quantile, $90 \%$ quantile, and maximum. The eighth value is the number of models or climate change scenarios used in each propertyregion combination (sample sizes). The combination of four properties, seven regions, and eight values creates the 224 numbers from K2020 that we use. Figure 1a (similar to Fig. 1b from K2020) shows results for changes in TC frequency, Fig. 1b (similar to Fig. 2b in K2020) shows results for changes in frequency of very intense TCs, Fig. 1c (similar to Fig. 3b in K2020) shows results for changes in the maximum intensity of TCs, and Fig. 1d (similar to Fig. 4b in K2020) shows results for changes in precipitation.

These data are affected by many sources of uncertainty. For instance, in K2020, the authors processed the data from the different studies in such a way as to be able to present the results for the same common $2^{\circ} \mathrm{C}$ change. This processing involves making assumptions about the TC response to climate change and its relation to GMST. Also, the set of studies used in K2020 is an ensemble of opportunity and was not created in any formal statistical way. In addition, the individual studies themselves are affected by many uncertainties, and are based on numerical models that do not represent TCs fully realistically. In the worst case, it is possible that none of the models come close to representing the true climate change signal. 
Furthermore, the authors of the individual studies made various assumptions in order to convert the output from their models into estimates of future TC numbers. For example, different procedures were used to identify modeled tropical storms in the different studies. Also, some studies reported rainfall rates for an averaging radius or radii that did not include $150 \mathrm{~km}$, in which case the rate for the radius closest to $150 \mathrm{~km}$ was used by K2020. In addition, a few studies reported intensity changes in terms of central pressure that K2020 needed to approximately convert to a percent change in maximum wind speed. As a result of these and other uncertainties, the K2020 results should not be taken as precise predictions, although they could be considered as an attempt at creating a best estimate of the current state of knowledge of how TCs might change due to climate change.

The part of the uncertainty that is captured by using different models to estimate the changes in TC properties can be quantified by the ranges of results given in K2020, which we refer to as the model spread. For instance, in Fig. 1a, the range of frequency changes for TCs in the North Atlantic extends from below $-60 \%$ to above $+100 \%$. The levels of model spread vary significantly by variable being considered. In comparing the panels in Fig. 1, we can see that estimates for changes in the frequency of TCs and the frequency of very intense TCs show the highest model spread. For the frequency of TCs, although there are more projections that show a decrease in frequency than an increase, there are still many that show increases, and the interquartile range overlaps zero for most of the regions. For the frequency of intense TCs, although there are more projections that show an increase than a decrease, there are still many that show decreases, and again the interquartile range overlaps zero in most regions. However, K2020 noted that the projections of decreased intense TC frequency tended to come from the relatively lower resolution modeling studies, in which one might have lower confidence. The projections for intensity and rainfall rate show lower model spread than the projections for frequency. Most models considered in K2020 show increases in intensity, and most models show increases in rainfall rate. Increases in intensity and rainfall rate are also predicted by fundamental physical arguments (see the discussion in K2020), in a way that changes in frequency are not.

Further insight into the relative sizes of the model uncertainties of the different projections can be derived from the sample sizes. These are given by the numbers below the horizontal axes of Fig. 1. The estimates of TC frequency are calculated from, on average across the seven regions, 128 models and scenarios; the estimates of frequency of very intense TCs are calculated from, on average, 51 models and scenarios; the estimates of intensity are calculated from, on average, 22 models and scenarios; and the estimates of precipitation are calculated from, on average, 17 models and scenarios. Since the changes in intensity and precipitation are based on fewer models, they should be treated with more caution. If more models were used, there is no way to know whether the statistical metrics $(5 \%$ or $10 \%, 25 \%$, median, $75 \%$, and $90 \%$ or $95 \%$ ) would increase or decrease, but the minimum and maximum would likely become more extreme.

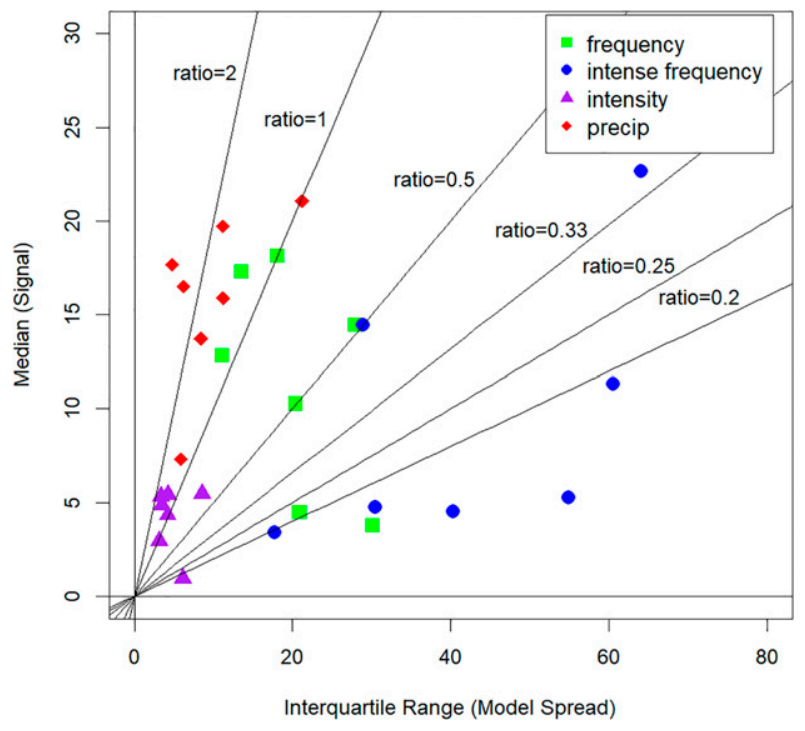

FIG. 2. From the data in Fig. 1, the median changes, or "signal" for four TC properties and seven regions (vertical axis) vs interquartile ranges, or "spread" (horizontal axis). The diagonal lines show lines of constant ratio of signal to spread.

Figure 2 illustrates the variations in model spread in the various results from K2020 by plotting the signal of change, defined here using the absolute value of the median change, against the model spread, defined here using the interquartile range of the change. The six diagonal lines show different levels of signal-to-spread ratio. We see that the changes in precipitation show the highest signal-to-spread ratios, followed by changes in intensity. For the change in precipitation, the signal is equal to or greater than the spread in all cases. The frequency of storms shows lower signal-to-spread ratios, and the frequency of very intense storms shows the lowest ratios. For the frequency of very intense storms, the spread is more than 5 times the signal for five out of seven cases. This emphasizes that caution needs to be exercised when interpreting the median frequency changes, and that the median values should not be used without also considering the spread, especially for frequency and the frequency of very intense storms.

A limitation of the K2020 results is that they do not cover all aspects of TCs that might change due to climate change. For instance, it has been suggested that the translation speed of TCs might change due to climate change (Kossin 2018), and that latitude of lifetime maximum intensity might change (Kossin et al. 2014). These phenomena are discussed in the text of K2020, but no numerical results are given. Also, although K2020 do include intensity change, where intensity is measured using maximum wind speed, there are reasons why measuring intensity using central pressure may be more useful for risk models (Klotzbach et al. 2020).

\section{b. GMST data}

Since K2020 present changes corresponding to a $2^{\circ} \mathrm{C}$ increase in GMST, we will require past and future GMST data to convert the K2020 results into changes relative to specific 
temporal baselines and for the specific points in time at which projections are required. The past GMST data we use consist of annual values from NASA GISS (NASA 2021; Hansen et al. 2010) and extend from 1880 to 2019 . We have adjusted the data with a constant offset so that the period 1950-2019 has a mean of zero. The level of this offset has no impact on our analysis since our methods and results will only depend on changes in GMST, but using this particular period to derive the offset will prove useful for the interpretation of the results below. The 1950-2019 baseline is not the same as the standard preindustrial baseline often used to calculate changes in global temperature due to climate change. Changes in GMST relative to the 1950-2019 baseline will be considerably smaller than changes relative to the preindustrial baseline.

The future GMST data we use correspond to the four standard RCPs $(2.6,4.5,6.0$, and 8.5). The values are averages of output from the ensemble of climate models from the CMIP5 project (Taylor et al. 2012) and were downloaded from the KNMI data explorer (KNMI 2021). They run from 2006 to 2100 .

We have used GMST based on CMIP5 RCPs rather than the more recent CMIP6 SSPs (Eyring et al. 2016; Meinshausen et al. 2020) for our calculations because the risk modeling community is still at this point using RCPs to define future climate scenarios. However, future GMST values based on the CMIP6 SSPs are also included in the software tools we provide. The CMIP6 GMST values are averages of output from an ensemble of climate models from the CMIP6 project and were also downloaded from the KNMI data explorer.

To concatenate the historical GMST data and the CMIP5 and CMIP6 GMST data, we adjust the CMIP5 and CMIP6 data with a constant offset, separately for each RCP and SSP, so that during the 14-yr overlap period from 2006 to 2019 the datasets have the same mean. The adjusted CMIP5 and CMIP6 data are only used from 2020 onward.

There are two somewhat arbitrary choices that have been made in our selection of GMST data. First, we have picked just one of several possible sources of historical GMST data: other sources include Morice et al. (2021) and Vose et al. (2012). However, these different datasets contain similar values, and using a different dataset would likely not affect our results to any material extent. Second, we have used a straight average of all the CMIP5 models. Alternative strategies would be to eliminate some of the less-well-performing models or to apply weights to the models. Again, this would likely not affect our results to any material extent.

Figure 3a shows the concatenated historical and CMIP5 GMST data that we use, with the historical observed data in black and the RCPs in color. Levels of $0^{\circ}$ and $2^{\circ} \mathrm{C}$ are marked by horizontal lines. Only RCP6.0 and RCP8.5 GMSTs reach $2^{\circ} \mathrm{C}$ relative to our $1950-2019$ baseline during this period, and the points in time at which they reach $2^{\circ} \mathrm{C}$, which are 2055 and 2079, are shown by vertical dot-dashed lines.

\section{Risk model baselines}

We now briefly review how risk models are constructed and give some examples of the temporal baselines used in TC risk modeling studies. We only include peer-reviewed and publically available results. We are aware that several more TC risk models exist, particularly in the private sector, but we have no access to information about them.

\section{TC risk models}

The purpose of TC risk models is to give estimates of the various risks due to tropical cyclones. They originated in the insurance industry in the 1960s (Friedman 1972), and since then, most work has gone into developing models for the risks that the insurance industry faces: property risks (as opposed to, e.g., infrastructure and life and health risks) in heavily insured regions such North America, the Caribbean Sea, Japan, and Australia. Following Friedman (1972) the models are typically constructed of four parts: a hazard component that models TC tracks and wind speeds (and possibly rainfall and flooding) in a very large ensemble of many thousands of members, an exposure component that lists the buildings to be analyzed, a vulnerability component that models the damage done to different types of building as a function of hazard severity, and a financial model component that applies financial contracts to the results to calculate appropriate financial metrics. The most commonly discussed metrics are average annual loss, loss occurrence exceedance probability (the distribution of maximum loss in a year), and loss annual exceedance probability (the distribution of total loss in a year). These metrics can be calculated either for individual buildings or portfolios of buildings. In this study, we are considering the possibility of using the K2020 results to adjust the hazard component of these models, which would then lead to changes in these loss metrics.

To apply the K2020 results to a risk model, it is necessary to understand the baseline period of historical data used to build the model, as discussed in the introduction. For North Atlantic Ocean wind risk modeling, Vickery et al. (2000) calibrated their risk model using observed U.S. landfalling data from 1886 to 1996. Elsner and Bossak (2001) used U.S. landfalling data from 1851 to 2000, Emanuel et al. (2006) used Atlantic basin track and intensity data from 1970 onward, and Hall and Jewson (2007) used Atlantic basin track and intensity data from 1950 to 2003.

For northwest Pacific wind risk modeling, Yonekura and Hall (2011) used basin track and intensity data from 1945 to 2007. For northern Indian Ocean wind risk modeling, Asaduzzaman and Latif (2014) used landfalling data from 1877 to 2009. For southwest Pacific wind risk modeling, James and Mason (2005) used basin track and intensity data from 1969 to 2003, whereas for modeling risks in Australia due to TCs from both the southwest Pacific and southern Indian Oceans, Arthur (2021) used basin track and intensity data from 1981 to 2016, but other baselines for other variables. Less work has been done on TC rainfall modeling than on wind risk modeling. Grieser and Jewson (2012) built a rainfall risk model for TCs using rainfall observations from 1948 to 2007, Zhu et al. (2013) built a rainfall risk model for hurricanes in Texas using reanalysis data from 1980 to 2010, and Feldmann et al. (2019) built a model using rainfall observations from 1995 to 2016.

Presumably all these models would be updated to use the latest data if they were rebuilt today. We see that there is a 
(a) CMIP5 Global Mean Surface Temperature Projections

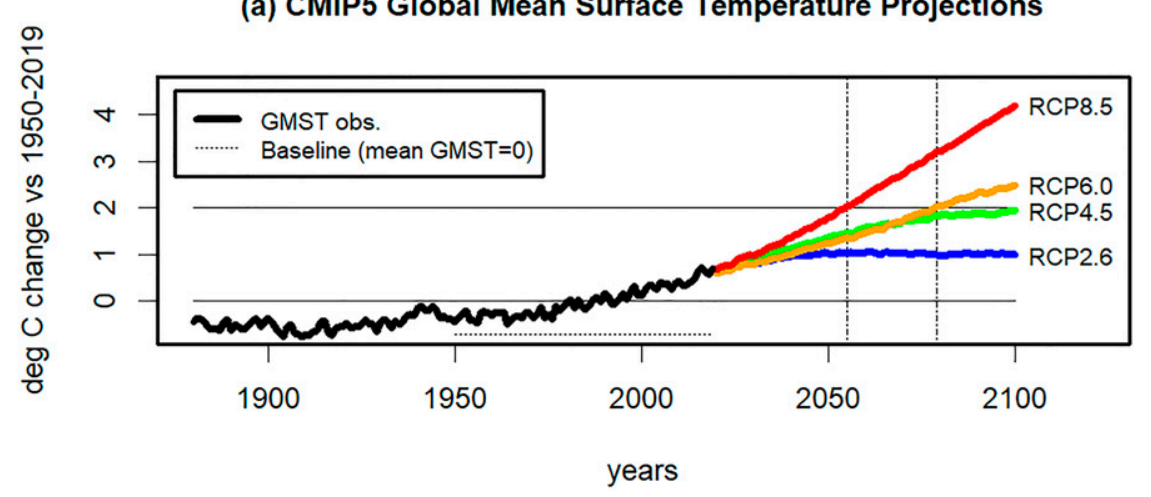

(b) Global TC Median Intensity Change Projections

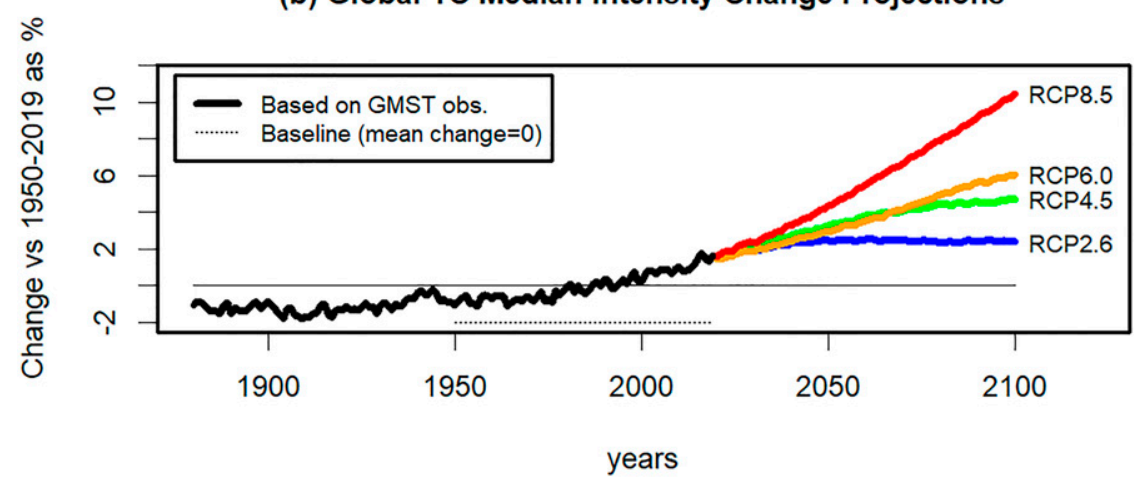

FIG. 3. (a) GMST observations (up to 2019) and projections derived from the CMIP5 ensemble (from 2020 to 2100). The observations are adjusted so that the mean from 1950 to 2019 is zero. This time period is shown as the dotted horizontal line. The two solid horizontal lines show changes of $0^{\circ}$ and $2^{\circ} \mathrm{C}$ relative to this baseline. The CMIP5 results for each RCP are adjusted so that the mean from 2006 to 2019 is equal to the mean of the observations. The two vertical lines show the years in which GMST for RCP6.0 and RCP8.5 reach $2^{\circ} \mathrm{C}$. (b) Global TC median intensity projections, derived from the GMST values in (a), using the model described in the text, which is fitted to the K2020 results shown in Fig. 1.

wide variety of different starting points used for the historical baselines, reflecting assessments of different levels of accuracy of data from different time periods (e.g., in the early twentieth century, open-ocean data are generally considered to be less accurate than landfalling data) and different levels of accuracy of data in different regions (e.g., the North Atlantic basin is observed in more detail than other basins). Different modelers have most likely also taken different views with regard to the best trade-off between using more data, which gives more precise estimates, and using more recent data, which is more accurate. Based on the above, we will use the period from 1950 to 2019 as a representative historical baseline but will also show results for 1900-2019 and 1970-2019 for comparison.

\section{Methods}

K2020 presented all of their results as changes in TC behavior corresponding to a $2^{\circ} \mathrm{C}$ increase in GMST, and by doing so did not need to specify a time period over which the changes would occur. The implication is that the same changes in TC behavior would occur whatever the time period, and that TCs will change more rapidly if GMST changes more rapidly, as it does in RCP8.5 relative to RCP2.6, for instance (see Fig. 3a). This assumed relationship between GMST and TC behavior is undoubtedly not correct at some level, but seems a reasonable approximation to make given the current state of understanding of TC behavior, pending further research.

The mathematical implication of presenting the changes in TC properties in this way is that these properties can be modeled as a function of GMST in order to interpolate and extrapolate them to the required time period. A simple model one might consider for the relationship between GMST $T_{i}$ and TC properties $F_{i}$, both in year $i$, would be a linear model for fractional changes in $F_{i}$ between years $m$ and $n$ of the form

$$
\frac{F_{n}-F_{m}}{F_{m}}=\beta\left(T_{n}-T_{m}\right),
$$

where $\beta$ is a parameter that represents a fractional change due to a change of GMST of $1^{\circ} \mathrm{C}$ and that could therefore be calculated as 0.5 times the values given in K2020 (with appropriate conversion between percent changes and fractional changes). 
However, the four TC properties considered in K2020 must always be nonnegative, and this model implies negative values of $F_{i}$ for some values of $T_{n}-T_{m}$. For example, the minimum value for North Atlantic frequency changes in K2020 is less than $-50 \%$ (Fig. 1a). When combined with temperature changes of $4^{\circ} \mathrm{C}$, as are seen in RCP8.5 in 2100 (Fig. 3a) this gives frequency changes of less than $-100 \%$, which leads to negative frequencies, which is nonsensical. To avoid this problem, we use the natural logarithm of the property $F_{i}$, which can be positive or negative, and write

$$
\ln F_{i}=\alpha+\beta T_{i} \quad \text { or },
$$

equivalently,

$$
F_{i}=e^{\alpha+\beta T_{i}}
$$

and this is the model for annual TC properties that we will use for all of our analysis below. K2020 provide fractional changes in properties (as percentages), and we will produce interpolated and extrapolated fractional changes from this model as follows. If $T$ changes from $T_{m}$ to $T_{n}$ and $F$ changes from $F_{m}$ to $F_{n}$, then the fractional change in $F$ implied by Eq. (3) is given by

$$
\frac{F_{n}-F_{m}}{F_{m}}=\frac{e^{\alpha+\beta T_{n}}-e^{\alpha+\beta T_{m}}}{e^{\alpha+\beta T_{m}}}=\frac{e^{\beta T_{n}}-e^{\beta T_{m}}}{e^{\beta T_{m}}}=e^{\beta\left(T_{n}-T_{m}\right)}-1,
$$

and we see that the parameter $\alpha$, which is a constant related to the absolute value of the property $F_{i}$, has dropped from this expression.

K2020 give fractional changes corresponding to $T_{n}-T_{m}=$ $2^{\circ} \mathrm{C}$. Writing the fractional changes given in $\mathrm{K} 2020$ as $K$ and substituting $T_{n}-T_{m}=2^{\circ} \mathrm{C}$ and $K$ into Eq. (4) give

$$
K=e^{2 \beta}-1,
$$

which can be solved for $\beta$ to give

$$
\beta=0.5 \ln (K+1) .
$$

Substituting this back into Eq. (4) gives

$$
\frac{F_{n}-F_{m}}{F_{m}}=e^{0.5\left(T_{n}-T_{m}\right) \ln (K+1)}-1 .
$$

This equation gives the fractional changes in $F$ from one year to another year as a function of the GMST change. For small values of $K$ and $T_{n}-T_{m}$, linearization of this equation gives

$$
\frac{F_{n}-F_{m}}{F_{m}} \approx 0.5\left(T_{n}-T_{m}\right) K,
$$

which is the linear equation given in Eq. (1), although we will use Eq. (7) to avoid the negative values of $F$ that can arise with this linear equation, as discussed above.

To apply the K2020 results to risk models we need to calculate the fractional changes between TC properties for a risk model baseline that runs over the $N_{1}$-yr period $P_{1}$ and a projection that runs over the $N_{2}$-yr period $P_{2}$. The mean of $F$ over a block of $N_{j}$ years $P_{j}$ is given by $M_{j}=\left(1 / N_{j}\right) \sum_{i \in P_{j}} F\left(T_{i}\right)$, and the fractional change in $M$ between the means $M_{1}$ and $M_{2}$ of periods $P_{1}$ and $P_{2}$ is given by $\left(M_{2}-M_{1}\right) / M_{1}$. Combining with Eq. (3) gives

$$
\begin{aligned}
\frac{M_{2}-M_{1}}{M_{1}}= & \frac{\frac{1}{N_{2}} \sum_{i \in P_{2}} F\left(T_{i}\right)-\frac{1}{N_{1}} \sum_{\in P_{1}} F\left(T_{i}\right)}{\frac{1}{N_{1}} \sum_{i \in P_{1}} F\left(T_{i}\right)}, \\
= & \frac{\frac{1}{N_{2}} \sum_{i \in P_{2}} e^{\alpha+\beta T_{i}}-\frac{1}{N_{1}} \sum_{i \in P_{1}} e^{\alpha+\beta T_{i}}}{\frac{1}{N_{1}} \sum_{1} e^{\alpha+\beta T_{i}}}, \\
= & \frac{\frac{1}{N_{2}} \sum_{i \in P_{2}} e^{\beta T_{i}}-\frac{1}{N_{1}} \sum_{i \in P_{1}} e^{\beta T_{i}}}{\frac{1}{N_{1}} \sum_{i \in P_{1}} e^{\beta T_{i}}} .
\end{aligned}
$$

If the periods $P_{1}$ and $P_{2}$ both only contain 1 year, then this simplifies to Eq. (4). This is the expression that we will use to derive our adjusted projections, as a function of the definition of the baseline $P_{1}$ and the projection period $P_{2}$. For the projection period $P_{2}$ we will initially use single years, but since there is a small amount of interannual variability in the GMST data (see Fig. 3a) we will also use 11-yr periods, centered on the single years of interest, to smooth the estimates.

For clarity, we note that the sequence of calculations we are using in Eq. (11) is as follows: (i) we transform annual GMST values using $e^{\beta T_{i}}$, (ii) we calculate the average of these transformed values over the two time periods, and (iii) we calculate the fractional change in the averages. Because of the linearity of the calculation of fractional changes, this would be equivalent to (i) transforming annual GMST values using $e^{\beta T_{i}}$, (ii) calculating the average of these transformed values over the baseline time period only, (iii) calculating the fractional change for every individual year in the projection time period, and (iv) calculating the average of these fractional changes. In other words, it makes no difference whether the fractional change is calculated before or after averaging over the projection time period.

However, because $e^{\beta T_{i}}$ is a nonlinear transform, neither of these sequences of calculations are equivalent to (i) calculating the average of the GMST values over the two time periods, (ii) transforming the average GMST using $e^{\beta T}$ where $T$ is now the average GMST, and (iii) calculating the difference in the transformed values. We will not use this calculation. In summary: we transform annual GMST using the exponential before we average over time, in order to be completely consistent with our model for how the TC properties vary as a function of GMST on a yearly basis [Eq. (3)] and to be consistent with the idea that we are deriving fractional changes that will be applied to a risk model that is based on a multiyear baseline average of TC behavior, given by $\left(1 / N_{1}\right) \sum_{i \in P_{1}} e^{\alpha+\beta T_{i}}$ in our model.

\section{Uncertainty around the GMST values}

Both the historical GMST values, and the future GMST values, are uncertain. For the historical values this uncertainty could be quantified by comparing different datasets and 
considering observational uncertainties. For the future GMST values this uncertainty could be quantified from the spread across the CMIP5 models. However, the derivations above ignore this uncertainty. This is deliberate: adding uncertainty to Eq. (3) would be possible, but more complex, and given the various other uncertainties in the whole analysis, as discussed in section $2 \mathrm{a}$ above, the additional complexity would not seem to justify the benefit. Equation (11) is an equation that can be computed with a few lines of computer code and simple data inputs, which is, we feel, exactly the right level of sophistication for practical solutions to the problem of how to adjust the K2020 results to a format that can be used in risk models. In addition, GMST uncertainty was not taken into account in K2020 when the projections for different models were combined to give the input data that we are using, and so incorporating it now would create an inconsistency.

\section{Results}

We now show various results calculated using Eqs. (6) and (11), where the parameter $\beta$ is determined for each of the 196 metrics from the data from K2020 as shown in Fig. 1. There are many possible combinations of choice of baseline, projection time, variable, region, and RCP. The results we show are a subset of the kinds of results that can be generated, chosen to illustrate the method and some key concepts. For three of our examples, we choose a single RCP. Based on a subjective evaluation of the discussion in Sanford et al. (2014), Harvey (2020) and the first UNEP synthesis report (Baste and Watson 2021) we conclude that RCP6.0 is currently the most likely future scenario, and so when we only consider one RCP, we use RCP6.0.

\section{a. Median global intensity for four RCPs}

One way to present results from the conversion model described in section 4 is to fix the baseline, variable, and region; vary the projection time; and show results for the median value for different RCPs. Applying this idea, Fig. 3b shows times series of annual values of the fractional change $\left(M_{2}-M_{1}\right) / M_{1}$ as percentages, calculated using Eq. (11), for the median value of global TC intensity, where $P_{1}$ corresponds to our representative historical baseline 1950-2019 and $P_{2}$ corresponds to individual years from 1880 to 2100 . The median value of global TC intensity has a value of $K$ of 0.049 , giving a value for $\beta$ of 0.024 . In a linearized sense, this corresponds to a $2.4 \%$ change in intensity for every degree Celsius. Figure $3 \mathrm{~b}$ shows that the fractional changes in intensity produced by Eq. (11) follow the shape of the GMST curves shown in Fig. 3a very closely. The model adjusts the K2020 results in such a way that there is a zero mean intensity change for the historical baseline 1950-2019 (marked by a horizontal dotted line on the plot) and an intensity change of $4.9 \%$ when the GMST for RCP6.0 and RCP8.5 reaches $2^{\circ} \mathrm{C}$ higher than during the historical baseline. In this way the intensity changes are consistent with a risk model built on a 1950-2019 baseline, and also consistent with the changes given in K2020. The shape of the curves in Fig. 3b is not exactly the same as the shape of the curves in Fig. 3a: because of

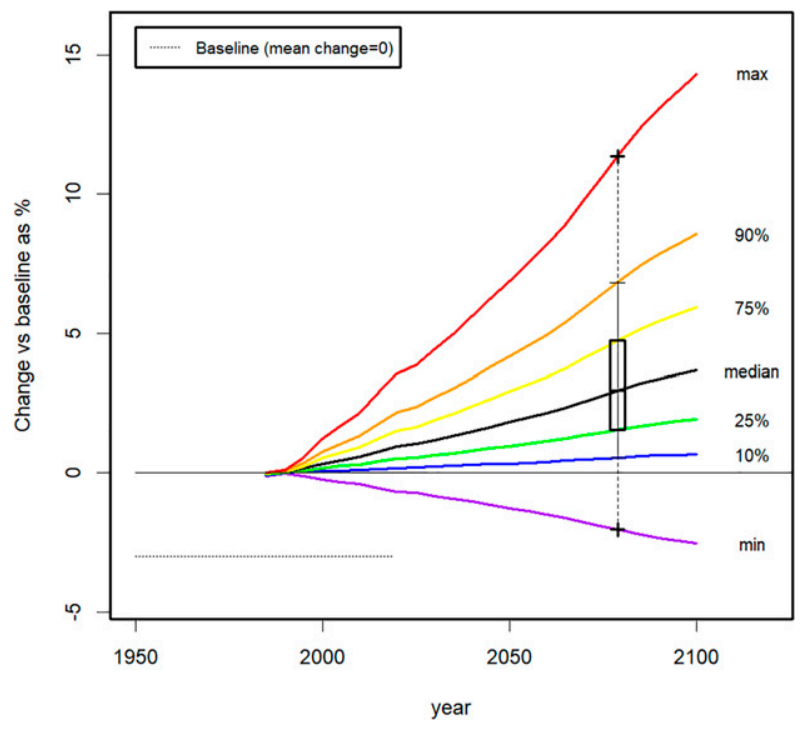

FIG. 4. North Atlantic TC intensity change projections based on RCP6.0. All values are relative to the average projection from the 1950 to 2019 baseline. The years of this baseline are shown by the horizontal dotted line. The seven colored lines show the distribution of possible changes. The box-and-whiskers plot comes from Fig. 1, and the lines are derived from the values illustrated by the box-and-whiskers plot using the GMST values shown in Fig. 3a and the interpolation and extrapolation model described in the text.

the use of the exponential in Eq. (3), variations are relatively smaller for lower values of GMST in Fig. $3 b$.

Figure $3 \mathrm{~b}$ shows that the combination of using a risk model based on a historical baseline, increasing GMST values during the historical baseline period, and the assumption that the TC intensity is related to the GMST, together lead to the implication that there may already have been a change in TC intensities by the end of the baseline period in 2019, relative to the mean TC intensities during the baseline period. However, estimating 2019 tropical cyclone climate in this way neglects the effects of climate variability, whether internal variability or forced variability. In particular, it neglects the effects of variations in aerosol concentrations, which may have had a larger impact on recent tropical cyclone climate than variations in greenhouse gases (Dunstone et al. 2013; Sobel et al. 2016, 2019b). As a result, these estimates of 2019 TC climate may be biased. For the North Atlantic, where tropical cyclone behavior has shown large decadal variability over the last half-century, statistical methods are sometimes used to attempt to adjust hurricane climate estimates for decadal time-scale variations in the historical data, which may be partly due to variations in aerosol concentrations (Jewson et al. 2009).

\section{b. North Atlantic intensity for RCP6.0}

A second way to present results from the conversion model is to fix the baseline, variable, region, and RCP; vary the projection time; and show results for all seven metrics from the distribution of model spread. Applying this idea, Fig. 4 


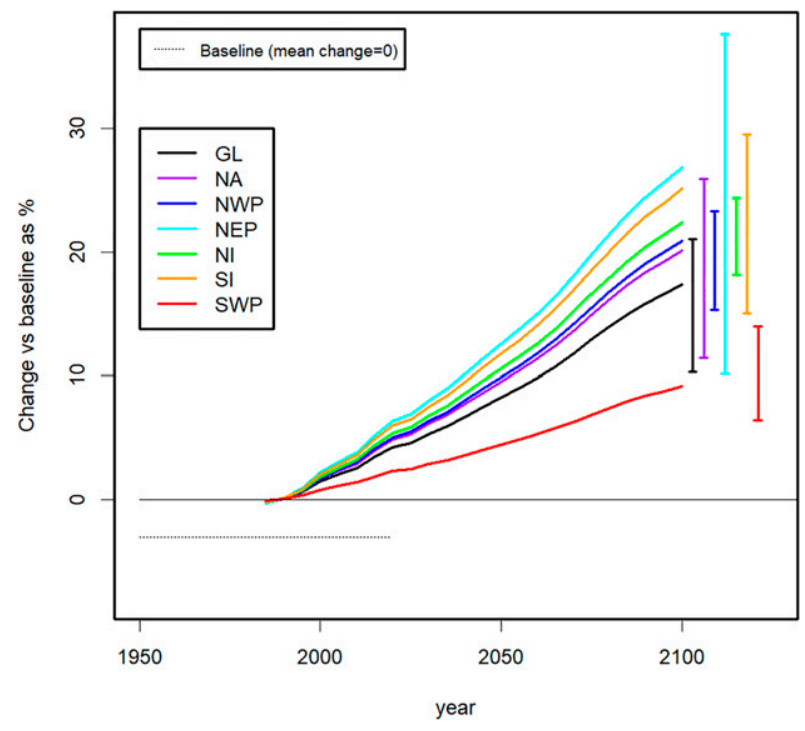

FIG. 5. Median projections for TC precipitation, relative to a 1950-2019 baseline, based on the model described in the text. The vertical lines show the interquartile ranges.

shows time series of the fractional change for all seven metrics, for the intensity of North Atlantic TCs, under RCP6.0. Figure 4 also shows the corresponding box-and-whiskers chart from Fig. 1c. In Fig. 3b, we showed annual values of median intensity change, calculated by setting the projection period $P_{2}$ in Eq. (11) to be one year long, while in this and subsequent figures we will show values smoothed in time by setting $P_{2}$ to be 11 years long. The fractional changes in Fig. 4 are zero near the middle of the baseline period, by definition, because they are fractional changes relative to the mean behavior during the baseline period. The zero point is not exactly in the middle of the baseline period because the mean TC properties during the baseline period are not exactly equal to the TC properties of the midyear of the baseline period, because of the variations in GMST during the baseline period and because of the exponential function used in Eq. (11). In Fig. 3b, we showed fractional changes for the whole time period back to 1880 , but in Figs. 4,5, and 6 we will only show it from this zero point onward. The values in Fig. 4 at the zero point are not exactly zero because the zero point may lie between years and because we are showing 11-yr averages.

We see clearly from Fig. 4 how our adjustment method interpolates between an assumption of zero change for the baseline period and the K2020 values given by the box-andwhiskers and then extrapolates into the future. The interpolation is not linear in time but follows the variability of the GMST during this period: the variability of observed GMST up to 2019, and the variability of CMIP5 modeled GMST for RCP6.0 from 2020 onward. In this particular case, since the evolution of GMST in RCP6.0 is close to linear in time over this time period, the evolution of the intensities is also close to linear in time. The evolution of GMSTs in RCP2.6 and RCP4.5 is not close to linear in time over this period and would not give variations in intensity that are close to linear in time. The

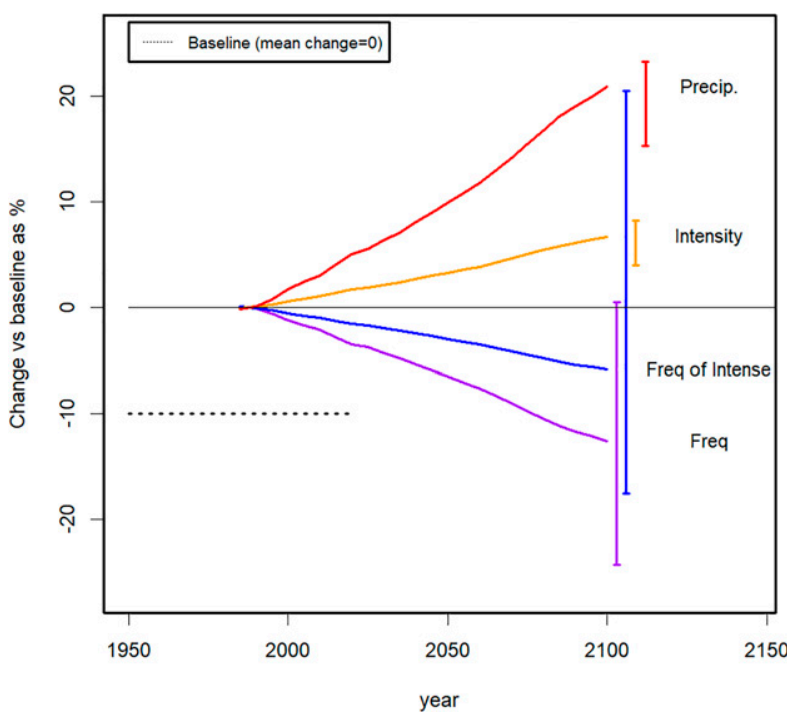

FIG. 6. Median projections for four different TC properties, for the NWP basin, relative to the 1950 to 2019 baseline. The vertical lines show interquartile ranges.

positive skew in the projected distribution of intensity, which can already be seen in Fig. 1c, is very clear in this figure.

\section{c. Median precipitation changes by basin for RCP6.0}

A third way to present results from the conversion model is to fix the baseline, variable, and RCP; vary the projection time; and show median results for every region, along with the interquartile range. Applying this idea, Fig. 5 shows time series of the fractional change for the median precipitation change, by basin, for RCP6.0, with interquartile ranges. We see a very wide range of fractional changes in precipitation across the basins, from the lowest changes in the SWP to the highest changes in the NEP. Some of the range may be due to undersampling, given the small number of models used for calculating these changes (see Fig. 1d).

\section{d. TC changes by variable for the North Atlantic RCP6.O}

A fourth way to present results from the conversion model is to fix the baseline, the region, and the RCP; vary the projection time; and show results for all variables for the median and interquartile range. Applying this idea, Fig. 6 shows time series of the fractional change for the median, by variable, for RCP6.0, for the northwest Pacific, along with interquartile ranges. We see decreases for both frequencies, and increases for precipitation and intensity, with the largest increases for precipitation. The skewness of some of the ranges is apparent.

\section{e. Impact of baseline}

A fifth way to present results from the conversion model is to fix the variable and region, vary the projection time, and show median results for different baselines and RCPs, and we apply this idea in Fig. 7. Figure 7 is similar to Fig. 3b, but now shows 11-yr smoothed results for three different baselines. Baseline 1 is 1900-2019, baseline 2 is 1950-2019 (as used in the previous figures), and baseline 3 is $1970-2019$. Baseline 1 shows the 


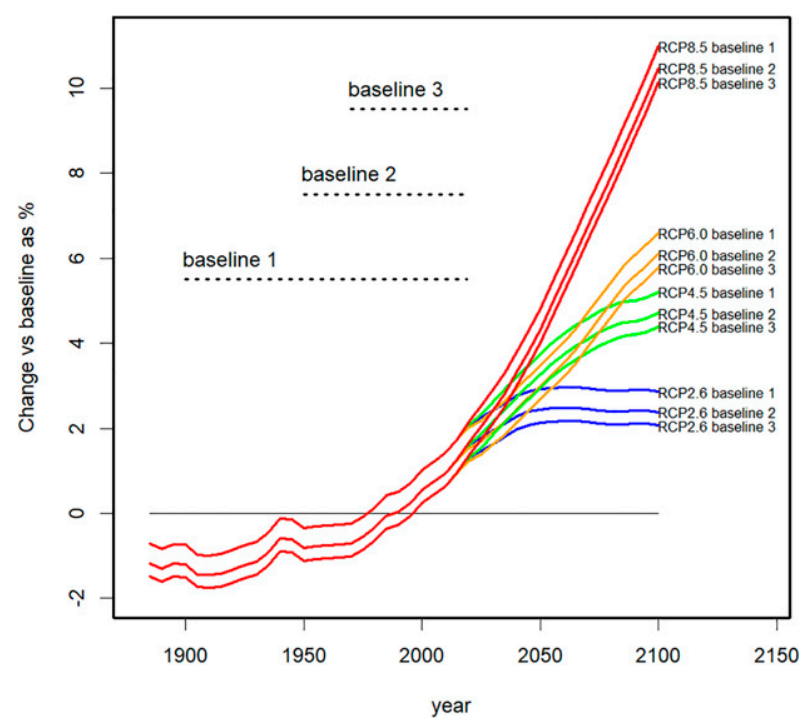

FIG. 7. Global TC intensity projections, for four RCPs, relative to three different baselines: baseline 1 (1900-2019), baseline 2 (1950-2019), and baseline 3 (1970-2019).

largest changes, simply because it includes earlier and hence cooler GMST data, leading to larger changes in GMST from the baseline to the projection period, and hence larger changes in intensity. We see that for the present day (i.e., 2021) the different RCPs give almost identical results, which reflects how we have constructed the GMST time series: the four RCP GMST time series are the same, by construction, up to and including 2019, and hence only deviate a very small amount by 2021. However, the three different baselines lead to very different results, already in 2021 . The difference due to different baselines then remains roughly constant over time. Farther in the future, for example, for 2100 , the main driver of the spread in the results becomes the difference between the different RCPs. We conclude that for quantifying the implied climate change so far, correct accounting for the baseline being used is more important than the choice of RCP. This emphasizes that users of risk models should make sure they understand the precise details of what baseline has been used to build the risk model they are using before they make any climate change adjustments. For this variable, for climate change in the midcentury (2030-70), correct accounting for the baseline and the choice of RCP are both important. In the far future (2100), correct accounting for the baseline is still important, but choice of RCP is more important.

\section{f. Implied climate change so far}

A sixth way to present results from the conversion model is to fix the baseline, projection time, and RCP and show results for all variables, regions, and metrics, following Fig. 1. Applying this idea, Fig. 8 shows projections for all variables and all regions, for the baseline 1950-2019 (the representative baseline, or baseline 2) for RCP6.0, and for an 11-yr period around the year 2020. The choice of RCP is in fact immaterial in this case, since in 2020, and the 11 years around 2020, the four RCPs give almost exactly the same results (see Figs. 3b and 7 and the discussion in the previous section). The choice of baseline is, however, material as we have seen in Fig. 7. Use of baseline 1 would give results farther from zero than those shown in Fig. 8, whereas use of baseline 3 would give results closer to zero. Because of the way our baseline adjustments work, Fig. 8 is essentially the same as Fig. 1, but with different values on the vertical axis. Since 2020 is essentially the present day, we refer to these projections as implied climate change so far.

Figure 8 shows median changes in implied climate change so far as follows: for frequency of all TCs, median changes ranging from $-90 \%$ to $-1 \%$ depending on the basin, with large model spread; for frequency of very intense TCs, median changes ranging from $-7 \%$ to $+9 \%$ depending on the basin, with large model spread; for TC intensity, changes of $0 \%-2.5 \%$ depending on the basin, with lower model spread, and for TC precipitation, changes of $3 \%-8.5 \%$ depending on the basin, with still lower model spread. As discussed in section 5 a above, these projections for 2020 only take into account the long-term climate change signal as extracted from climate model simulations, and ignore interannual, decadal and interdecadal variations in TC behavior. They are therefore not necessarily good estimates of the actual TC climate in 2020.

It is reasonable to ask whether these changes have already been observed. However, one would not necessarily expect to be able to detect long-term climate change signals of this size easily in recent observational data, because the size of the changes is small by comparison with the amplitude of internal variability [for papers on detecting trends in historical TC activity, see, e.g., Landsea et al. (2006), Elsner et al. (2008), Holland and Bruyere (2014), Knutson et al. (2019), Kossin et al. (2020), and Jewson and Lewis (2020)]. As a result, these changes are difficult to validate using observations. In addition, these results suffer from the uncertainties described in section $2 \mathrm{a}$.

\section{g. Climate change in 2100, and sources of uncertainty}

Figure 9 is similar to Fig. 8 but now shows projections of climate change for 2100 for RCP6.0. In addition, in colors, it shows the median changes for all four RCPs. In this way, we can compare the variations in the projections for 2100 due to variations in the K2020 results, which arise from the use of many different models (model spread), with the variations due to the choice of RCP. In Fig. 9a, we see that for frequency change the variations due to the choice of RCP are smaller than the variations due to model spread. In Fig. 9b, we see the same pattern. In Figs. 9c and $9 \mathrm{~d}$, the variations due to the choice of RCP are comparable to the model spread. We conclude that for intensity and precipitation the results are highly sensitive to the choice of RCP, whereas for frequency the choice of RCP is slightly less important because the results are dominated by model spread. Projections of frequency change for 2100 for different RCPs that ignore the model spread would be misleadingly precise. In other words, assuming that by using a range of RCPs one can capture most of the uncertainty would not be at all correct, because it would ignore the model spread, which is a large source of uncertainty.

\section{Conclusions}

A recent meta-analysis of the effects of climate change on various aspects of tropical cyclone behavior produced multimodel 
(a) TC Freq. Change (to 2020)

(b) Very Intense TC Freq. Change (to 2020)

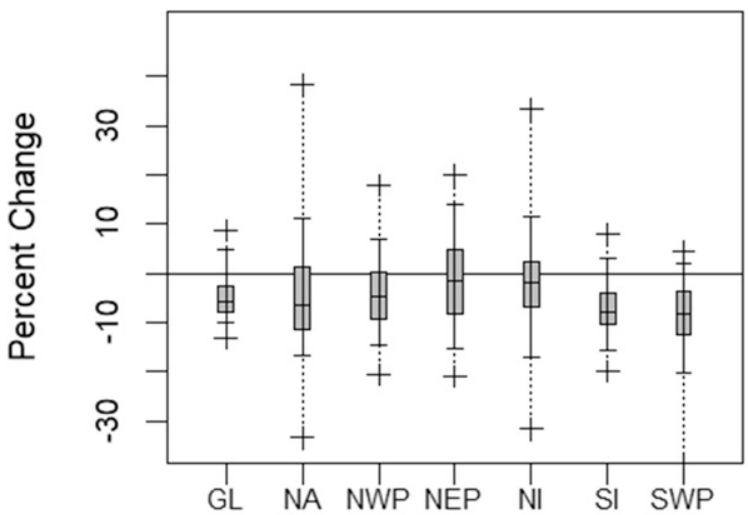

(c) TC Intensity Change (to 2020)

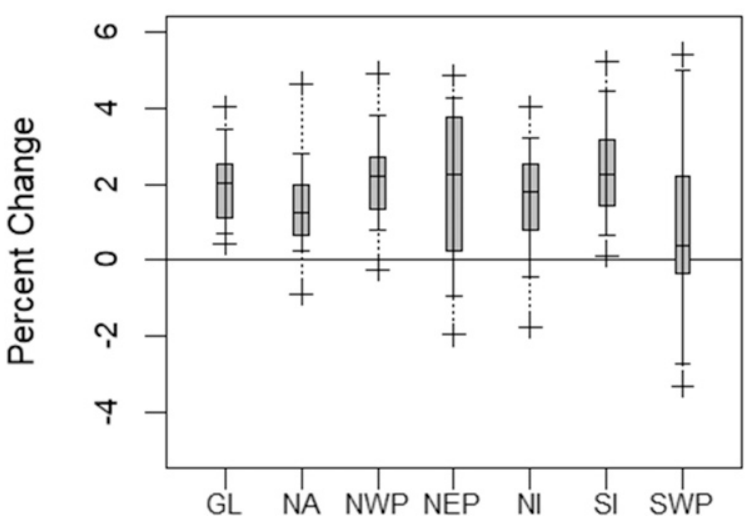

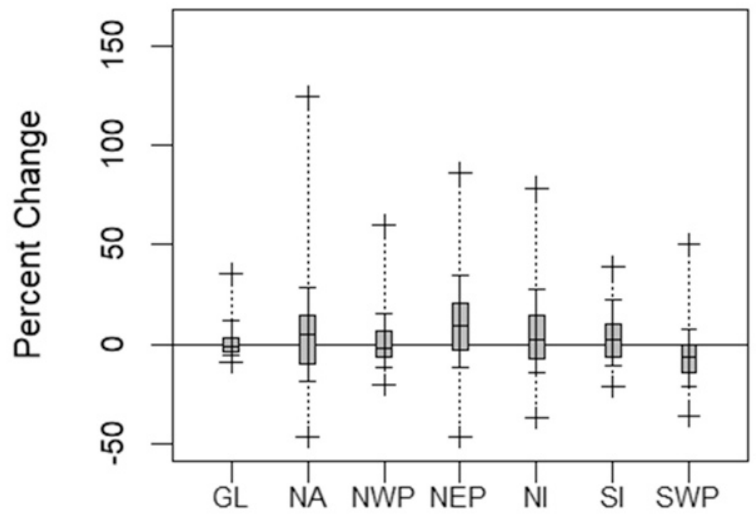

(d) TC Precip. Change (to 2020)

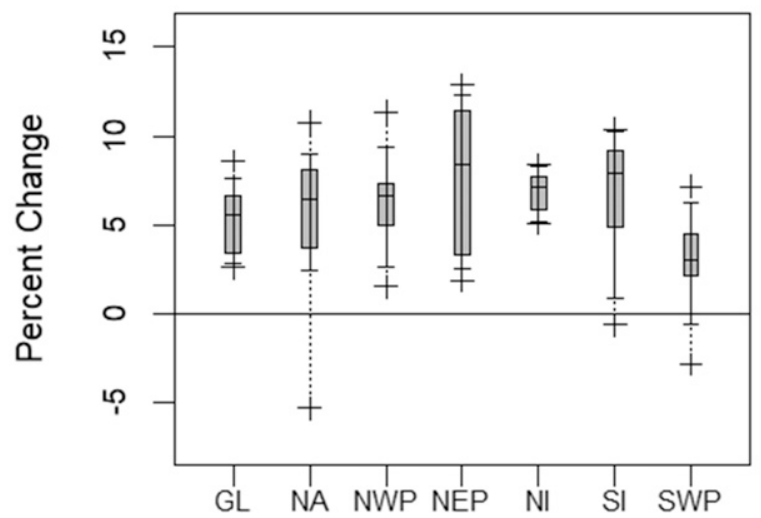

FIG. 8. As in Fig. 1, but now showing changes in TC properties for 2020 for RCP6.0, derived from the data shown in Fig. 1 by using the method described in the text.

multistudy projections of distributions of change for four tropical cyclone properties (K2020). The different studies were combined by expressing the projected changes in terms of the change corresponding to a $2^{\circ} \mathrm{C}$ increase in global mean surface temperature. The use of $2^{\circ} \mathrm{C}$ is a reasonable way to combine different studies and a concise way to communicate results. However, the results for $2^{\circ} \mathrm{C}$ of warming cannot be directly incorporated into risk models, which are typically based on observed data for certain historical periods.

We address the question of how to convert the K2020 results into a format that can be used in risk models that use a historical period baseline to determine the TC climate in the model. We start by explicitly defining a model for the relationship between GMST and TC properties. A linear model for fractional changes fails because, in some cases, it produces projections of percentage change that are below $-100 \%$. A log-linear model, in which the log of the TC property is a linear function of the GMST, resolves this problem. The log-linear model allows us to derive simple expressions that convert the projections from K2020 into percentage changes of TC properties for any temporal baseline and any projection period. For instance, K2020 state that, globally, the median change in TC rainfall rate corresponding to a warming of $2^{\circ} \mathrm{C}$ is $14 \%$. For a 1950-2019 historical data baseline, and RCP6.0 projections for 2020 and 2050, this translates into changes of $5.6 \%$ and $9.7 \%$, respectively.

We apply the log-linear model to the 196 TC changes from $\mathrm{K} 2020$, corresponding to four variables, seven basins, and seven values from the distribution created from the different models and studies that they consider. We show a number of examples. In particular we find the following three results: (i) The baseline used to make the adjustments has a large influence on the resulting projections, especially for climate change so far and midcentury projections. This emphasizes the need for users of TC risk models to understand what baseline the model they are using is based on, before considering making any adjustments to represent climate change. (ii) Relative to the typical historical baselines used in TC risk modeling, the K2020 results suggest that material changes in TC properties may have already occurred by 2021, in particular for rainfall. Future changes in TC properties, relative to the historical baseline, are then a combination of the change so far and the change going forward into the future. (iii) For projections of TC properties in 
(a) TC Freq. Change (to 2100)

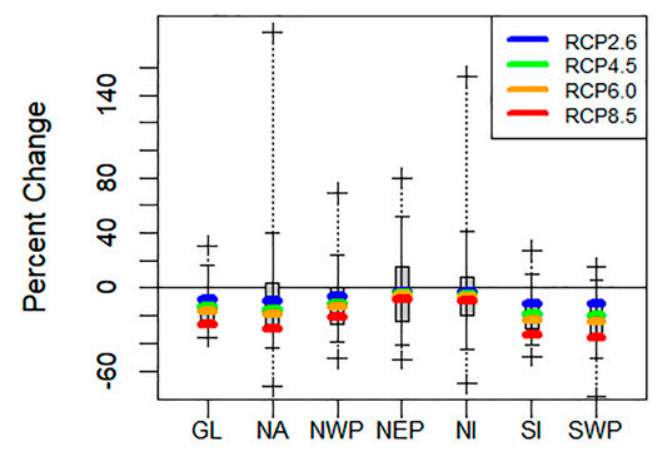

(c) TC Intensity Change (to 2100)

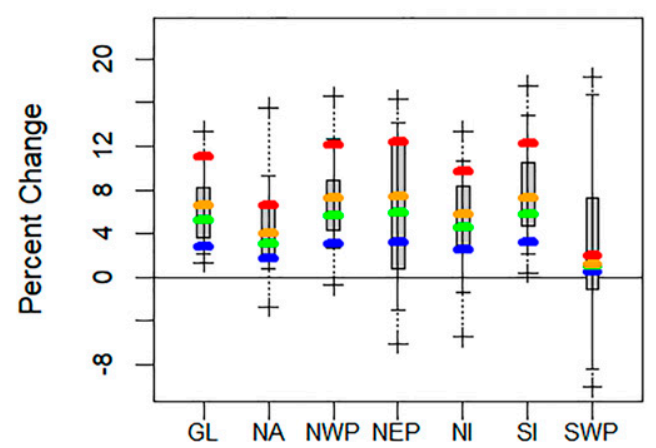

(b) Very Intense TC Freq. Change (to 2100)

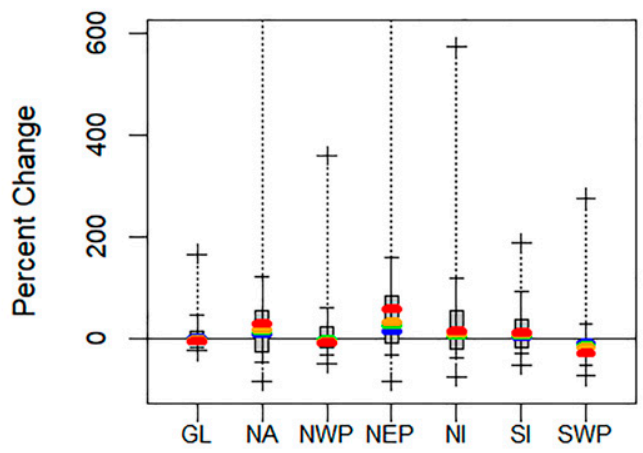

(d) TC Precip. Change (to 2100)

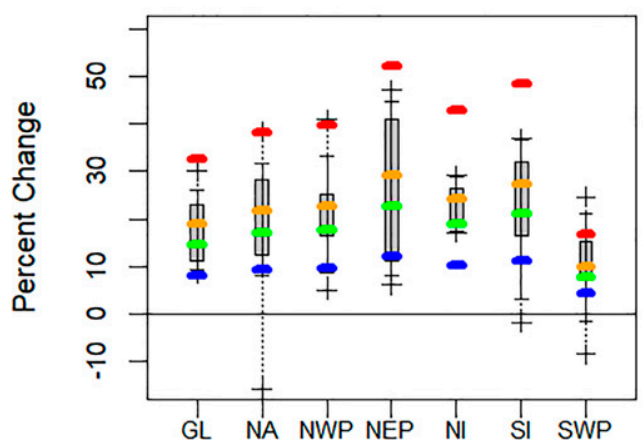

FIG. 9. As in Fig. 1, but now, in black, showing changes in TC properties for 2100 for RCP6.0, derived from the data shown in Fig. 1 by using the method described in the text. The colors show median changes for four RCPs: RCP2.6 (blue), RCP4.5 (green), RCP6.0 (orange), and RCP8.5 (red).

2100, model spread dominates over variations due to the choice of RCP for frequency projections. Model spread is also important for intensity and precipitation projections and is comparable in size to the size of variations in the projections due to the choice of RCP.

There are too many combinations of baseline, projection period, variable, region, and quantile for it to be practical to tabulate all possible results. We have therefore developed some simple software code that can be used to calculate the adjusted versions of the K2020 results for a user-specified baseline and projection time period. Three versions of the code have been developed. Two of the versions (in $\mathrm{R}$ and Python) require an appropriate interpreter and some limited editing of computer code to be able to adjust the input parameters. The third (in JavaScript) has a graphical user interface, requires nothing more than a browser to run, and works with any operating system. The computer codes are available online at Jewson et al. (2021b).

Further work is required before the results from our analyses would be ready to use to adjust risk models. One particular issue we have not addressed is that changes in frequency and intensity of TCs are not independent, and this would need to be accounted for in some appropriate way. Other issues, such as how to propagate the uncertainty in these results into a risk model framework and how to deal with mismatches between the variable definitions considered here and the variable definitions used in any particular risk model, would also have to be addressed. Nevertheless, we believe that the mathematical model and results, along with the accompanying data and software, will facilitate the incorporation of climate change information about TCs into risk models, which in turn will, it is hoped, lead to better management of the risks associated with TCs around the world.

Acknowledgments. The author thanks Tom Knutson for providing the data from $\mathrm{K} 2020$, for allowing the data to be made available in a repository and in the software tools, and for providing helpful feedback on an early version of the paper. We also thank NASA for making the GMST data available and Lighthill Research Network for funding this work.

Data availability statement. The GMST data are available from NASA (NASA 2021). The data from K2020 are available 
at Jewson et al. (2021a). Computer code used for some of the analysis will be available at Jewson et al. (2021b) on publication of the article.

\section{REFERENCES}

AOML, 2014: Hurricane database. NOAA/Hurricane Research Division, accessed 14 June 2021, https://www.aoml.noaa.gov/hrd/ hurdat.

Arthur, W., 2021: A statistical-parametric model of tropical cyclones for hazard assessment. Nat. Hazards Earth Syst. Sci., 21, 893-916, https://doi.org/10.5194/nhess-21-893-2021.

Asaduzzaman, M., and A. Latif, 2014: Forecasting tropical cyclones in Bangladesh: A Markov renewal approach. Computational Intelligence Techniques in Earth and Environmental Sciences. Springer, 129-140.

Baste, I. A., and R. T. Watson, 2021: Making peace with nature: A scientific blueprint to tackle the climate, biodiversity and pollution emergencies. UNEP Rep., 168 pp., https://wedocs.unep.org/ xmlui/bitstream/handle/20.500.11822/34948/MPN.pdf.

Dunstone, N. J., D. M. Smith, B. B. B. Booth, L. Hermanson, and R. Eade, 2013: Anthropogenic aerosol forcing of Atlantic tropical storms. Nat. Geosci., 6, 534-539, https://doi.org/ 10.1038/ngeo1854.

Elsner, J., and B. Bossak, 2001: Bayesian analysis of U.S. hurricane climate. J. Climate, 14, 4341-4350, https://doi.org/10.1175/ 1520-0442(2001)014<4341:BAOUSH >2.0.CO;2.

- J. Kossin, and T. Jagger, 2008: The increasing intensity of the strongest tropical cyclones. Nature, $\mathbf{4 5 5}, 92-95$, https://doi.org/ 10.1038/nature07234.

Emanuel, K., S. Ravela, E. Vivant, and C. Risi, 2006: A statistical deterministic approach to hurricane risk assessment. Bull. Amer. Meteor. Soc., 87, 299-314, https://doi.org/10.1175/ BAMS-87-3-299.

Eyring, V., S. Bony, G. A. Meehl, C. A. Senior, B. Stevens, R. J. Stouffer, and K. E. Taylor, 2016: Overview of the Coupled Model Intercomparison Project Phase 6 (CMIP6) experimental design and organization. Geosci. Model Dev., 9, 19371958, https://doi.org/10.5194/gmd-9-1937-2016.

Feldmann, M., K. Emanuel, L. Zhu, and U. Lohmann, 2019: Estimation of Atlantic tropical cyclone rainfall frequency in the United States. J. Appl. Meteor. Climatol., 58, 1853-1866, https://doi.org/10.1175/JAMC-D-19-0011.1.

Friedman, D., 1972: Insurance and the natural hazards. ASTIN Bull., 7, 4-58, https://doi.org/10.1017/S0515036100005699.

Grieser, J., and S. Jewson, 2012: The RMS TC-rain model. Meteor. Z., 21, 79-88, https://doi.org/10.1127/0941-2948/2012/0265.

Hall, T., and S. Jewson, 2007: Statistical modeling of North Atlantic tropical cyclone tracks. Tellus, 59A, 486-498, https://doi.org/ 10.1111/j.1600-0870.2007.00240.x.

$\longrightarrow$, and - 2008: Comparison of local and basin-wide methods for risk assessment of tropical cyclone. J. Appl. Meteor. Climatol., 47, 361-367, https://doi.org/10.1175/2007JAMC1720.1.

Hansen, J., R. Ruedy, M. Sato, and K. Lo, 2010: Global surface temperature change. Rev. Geophys., 48, RG4004, https:// doi.org/10.1029/2010RG000345.

Harvey, C., 2020: The worst climate scenarios may no longer be the most likely. Sci. Amer., 30 January 2020, https:// www.scientificamerican.com/article/the-worst-climate-scenariosmay-no-longer-be-the-most-likely/.

Holland, G., and C. Bruyere, 2014: Recent intense hurricane response to global climate change. Climate Dyn., 42, 617-627, https://doi.org/10.1007/s00382-013-1713-0.
James, M., and L. Mason, 2005: Synthetic tropical cyclone database. J. Waterw. Port Coastal Ocean Eng., 131, 181-192, https://doi.org/10.1061/(ASCE)0733-950X(2005)131:4(181).

Jewson, S., and N. Lewis, 2020: Statistical decomposition of the recent increase in the intensity of tropical storms. Oceans, $\mathbf{1}$, 311-325, https://doi.org/10.3390/oceans1040021.

_ , and Coauthors, 2009: 5 year prediction of the number of hurricanes which make US landfall. Hurricanes and Climate Change, J. Elsner and T. Jagger, Eds., Springer, 73-99.

- C. Barnes, S. Cusack, and E. Bellone, 2019: Adjusting catastrophe model ensembles using importance sampling, with application to damage estimation for varying levels of hurricane activity. Meteor. Appl., 27, e1839, https://doi.org/10.1002/met.1839.

— jections data. Zenodo, https://doi.org/10.5281/zenodo.4738906.

_ K. Kewson, and L. Jewson, 2021b: Knutson et al 2020 tropical cyclone projections baseline conversion codes. Zenodo, https:// doi.org/10.5281/zenodo.4739093.

Klotzbach, P., M. M. Bell, S. G. Bowen, E. J. Gibney, K. R. Knapp, and C. J. Schreck, III, 2020: Surface pressure a more skillful predictor of normalized hurricane damage than maximum sustained wind. Bull. Amer. Meteor. Soc., 101 (6), E830-E846, https://doi.org/10.1175/BAMS-D-19-0062.1.

KNMI, 2021: Climate explorer. Accessed 14 June 2021, http:// climexp.knmi.nl/start.cgi.

Knutson, T., and Coauthors, 2019: Tropical cyclones and climate change assessment. Part I: Detection and attribution. Bull. Amer. Meteor. Soc., 100, 1987-2007, https://doi.org/10.1175/BAMS-D-18-0189.1. , and Coauthors, 2020: Tropical cyclones and climate change assessment. Part II: Projected response to anthropogenic warming. Bull. Amer. Meteor. Soc., 101 (3), E303-E322, https://doi.org/10.1175/BAMS-D-18-0194.1.

Kossin, J., 2018: A global slowdown of tropical cyclone translation speed. Nature, 558, 104-107, https://doi.org/10.1038/s41586-018-0158-3.

_ K. Emanuel, and G. Vecchi, 2014: The poleward migration of the location of tropical cyclone maximum intensity. Nature, 509, 349-352, https://doi.org/10.1038/nature13278.

—, K. Knapp, T. Olander, and C. Velden, 2020: Global increase in major tropical cyclone exceedance probability over the past four decades. Proc. Natl. Acad. Sci. USA, 117, 11 975-11 980, https://doi.org/10.1073/pnas.1920849117.

Landsea, C., B. Harper, K. Hoarau, and J. Knaff, 2006: Can we detect trends in extreme tropical cyclones? Science, 313, 452454, https://doi.org/10.1126/science.1128448.

Lee, C., M. Tippett, A. Sobel, and S. Camargo, 2018: An environmentally forced tropical cyclone hazard model. J. Adv. Model. Earth Syst., 10, 223-241, https://doi.org/10.1002/2017MS001186.

Meinshausen, M., and Coauthors, 2011: The RCP greenhouse gas concentrations and their extensions from 1765 to 2300 . Climatic Change, 109, 213-241, https://doi.org/10.1007/s10584-011-0156-z.

- and Coauthors, 2020: The shared socio-economic pathway (SSP) greenhouse gas concentrations and their extensions to 2500. Geosci. Model Dev., 13, 3571-3605, https://doi.org/ 10.5194/gmd-13-3571-2020.

Morice, C., and Coauthors, 2021: An updated assessment of nearsurface temperature change from 1850: The HadCRUT5 data set. J. Geophys. Res. Atmos., 126, e2019JD032361, https:// doi.org/10.1029/2019JD032361.

NASA, 2021: Global temperature. Accessed 14 June 2021, https:// climate.nasa.gov/vital-signs/global-temperature/.

Sanford, T., P. Frumhoff, A. Luers, and J. Gulledge, 2014: The climate policy narrative for a dangerously warming world. Nat. Climate Change, 4, 164-166, https://doi.org/10.1038/nclimate2148. 
Sobel, A., S. J. Camargo, T. M. Hall, C.-Y. Lee, Michael K. Tippett, and A. A. Wing, 2016: Human influence on tropical cyclone intensity. Science, 353, 242-246, https://doi.org/10.1126/science.aaf6574.

C.-Y. Lee, S. J. Camargo, K. T. Mandli, K. A. Emanuel, P. Mukhopadhyay, and M. Mahakur, 2019a: Tropical cyclone hazard to Mumbai in the recent historical climate. Mon. Wea. Rev., 147, 2355-2366, https://doi.org/10.1175/MWR-D-18-0419.1. S. Camargo, and M. Previdi, 2019b: Aerosol versus greenhouse gas effects on tropical cyclone potential intensity and the hydrologic cycle. J. Climate, 32, 5511-5527, https://doi.org/ 10.1175/JCLI-D-18-0357.1.

Taylor, K., R. Stouffer, and G. Meehl, 2012: An overview of CMIP5 and the experiment design. Bull. Amer. Meteor. Soc., 93, 485498, https://doi.org/10.1175/BAMS-D-11-00094.1.
Vickery, P., P. Skerlj, and L. Twisdale, 2000: Simulation of hurricane risk in the US using empirical track model. J. Struct. Eng., 126, 1222-1237, https://doi.org/10.1061/ (ASCE)0733-9445(2000)126:10(1222).

Vose, R., D. Arndt, and V. Banzon, 2012: NOAA's Merged LandOcean Surface Temperature analysis. Bull. Amer. Meteor. Soc., 93, 1677-1685, https://doi.org/10.1175/BAMS-D-11-00241.1.

Yonekura, E., and T. Hall, 2011: A statistical model of tropical cyclone tracks in the western North Pacific with ENSOdependent cyclogenesis. J. Appl. Meteor. Climatol., 50, 17251739, https://doi.org/10.1175/2011JAMC2617.1.

Zhu, L., S. Quiring, and K. Emanuel, 2013: Estimating tropical cyclone precipitation risk in Texas. Geophys. Res. Lett., 40, 6225-6230, https://doi.org/10.1002/2013GL058284. 